Acetylcholinesterase inhibitors 4344

Acute intestinal pseudo-obstruction 4344

Acute leukemia 4348

Acute promyelocytic leukemia (relapse; treatment options) $\mathbf{4 3 7 3}$

Adjuvant therapy $\mathbf{2} 115, \mathbf{5} 429$

Adjuvant treatment $\mathbf{3} 227$

Adjuvant 5484

Advanced bladder carcinoma 4361, 5484

Advanced gastric cancer $\mathbf{3} 255$

Adverse events 6545

Aged patients 4355

Anaemia 180

Anal cancer 5456

Angiogenesis 3290

Antineoplastic agents 6539

Anxiety 151

Apoptosis 6581

Arsenic trioxide 4373

Assessment 166

Autologous peripheral blood progenitor cell support 4344

Autonomy 6524

B-cell lymphomas, cutaneous 4366

bcl-2 3234

Bexarotene 5477

Biliopancreatic neoplasms 4325

Bladder cancer 2147

Blood tests 3241

Blood transfusions 180

Bone metastasis 4341

Brain tumours 138

Breast 3277,6588

Breast cancer 127, 1 58, 2 115, 2 128, 3283,3290

Breast cancer, prognostic factors 5438

Breast cancer, metastatic 6578

\section{CA 19-9 5462}

Calcium folinate 5469

Cancer prevention $\mathbf{3} 283$

Capecitabine 2 161, 6578

Carboplatin 6568

Catheter occlusion 5469
CD20 4351

Cervical carcinoma 4334

Cervical carcinoma, locally advanced 5452

Cervix uteri 158

Chemoradiation 4325

Chemoresistance 3234

Chemotherapy $\mathbf{1}$ 63, 2 115, 2 120, 2161 , 2 167, 4 361, 5 429, 5 446, 5 484, 6539

Chemotherapy, weekly $\mathbf{6} 568$

Child 5489

Clinical research 6529

Clinical trial: randomized, multi-center, design, hypotheses, significance, sample size $\mathbf{6} 551$

CNS 4351

Colon cancer 3283

Colorectal cancer 1 44, 2 120, 3303

Combination therapy: gemcitabine, cisplatin 5462

Combined modality therapy $\mathbf{3} 295$

Coping 6557

Cutaneous lymphomas 4366

Cytarabine 4348

$\mathrm{DAB}_{389} \mathrm{IL}-2 \mathbf{5} 477$

Dendritic cells 3234

Depression 151

DNA ploidy 132

Docetaxel 163

Dose escalation $\mathbf{4} 325$

Double labelling 132

Drug resistance $\mathbf{6} 581$

Ductal carcinoma in situ, prognostic factors, diagnosis, therapy $\mathbf{6} 588$

Endocrine therapy $\mathbf{5} 429$

Endometrial cancer 5429

Endourology 4334

Epidermal growth factor receptor 3290

Error management $\mathbf{6} 545$

Error prevention strategies 6539

Erythropoietin 180

Esophageal cancer 2161

Esthesioneuroblastoma 3261

Estrogen 3290
Ethics 6524

Evidence-based medicine 5438

Ewing's Tumor 6573

Extremity 4341

5-FU 5469

F18-FDG PET 2155

F18-fluorodeoxy-D-2-glucose positron emission tomography $\mathbf{5} 489$

FACT-G 138

Fatigue (measurement) $\mathbf{1 5 1}$

Fatigue 2140

FDG-PET 5489

Fibroblast activation protein $\mathbf{1} 44$

FKV 6557

Flow cytometry 132

Follow-up 3303

Forceps biopsy 132

Freiburg Questionnaire Coping with Disease 6557

Gastric cancer 163

G-CSF 173

Gemcitabine 4325

General population 2140

GeneScan-analysis 5477

Geriatric assessment $\mathbf{4} 355$

Granulocytes 173

Guidelines 6535

Hashimoto's thyroiditis 3277

Head and neck neoplasms 6568

Hematemesis 3249

Hematuria 3249

Hemopoietic lymphoepithelial disease 121

HER2/neu 3261

Herceptin $^{\circledR} 2128$

High-dose chemotherapy 4344

High-dose radio/chemotherapy $\mathbf{6 5 7 3}$

Histamine 3234

Hodgkin's disease 5489

Human anti-human antibody 144

Hyperbilirubinemia $\mathbf{6} 578$

Hyper-lipasaemia 5473

Hyperthermia 2 120, 3295

IFN-a2b 2147

\title{
KARGER
}

(c) 2003 S. Karger GmbH, Freiburg 
Immunohistochemistry $\mathbf{3} 261$

Immunotherapy $\mathbf{3} 227$

Industrial sponsor, Interaction $\mathbf{6} 529$

Infection 173

Informed consent $\mathbf{6} 524$

Insulin-like growth factors $\mathbf{3} 290$

Interferon $\alpha \mathbf{3} 227$

Interleukin-2 $\mathbf{3} 227$

Intrapericardial instillation $\mathbf{2} 135$

Irradiation $\mathbf{2} 120$

Jaundice 6578

Local immunotherapy 2147

Longitudinal investigation $\mathbf{6} 557$

Lymph node dissection $\mathbf{6} 564$

Lymph node metastases $\mathbf{5} 456$

Lymph node ultrasound $\mathbf{3} 241$

Lymphoma 4351

Maintenance therapy 6573

Malignant melanoma 3241

Malignant pericardial effusion 2135

Malpractice 6535

MALT lymphoma 3277

Medical error 6545

Medication errors 6539

Melanoma 3 218, 3 227, 6581

Metastases 158

Metastasis 163,3218

Metastatic malignant melanoma 3249

Metastatic melanoma 3234

MFI 2140

Microvascular density 2147

Mitomycin C 2161

Mitoxantrone 2135

Modulation 2175

Monoclonal antibody 144

Monoclonality 5477

Mucosal metastasis 3249

Multidrug Resistance 2175

Multiple myeloma $\mathbf{1 8 0}$

Mycosis fungoides 4366

Myxoid liposarcoma 4341

Nasopharyngeal carcinoma $\mathbf{1} 12$

Neoadjuvant chemotherapy 127

Neoadjuvant 4361

Neuroectodermal tumors, differential diagnosis $\mathbf{3} 261$

Neutropenia 173
Nodular panniculitis 5473

Non-small cell lung cancer $\mathbf{3} 272$

Norm values 2140

Oesophageal carcinoma 132

Oncologic rehabilitation $\mathbf{1} 51$

Ovarian cancer 2 167, 5446

Oxaliplatin 3255

Paclitaxel 2 128, 6568

PAI-1 5438

Palliative therapy 5429

Pancreatic cancer 5462

Pancreatic carcinoma 5473

Patient safety 6545

Patterns of failure $\mathbf{1} 12$

Pelvic recurrence $\mathbf{5} 452$

Pericarditis 4348

PET diagnosis 2155

Pharmacokinetics 144

Physical activity $\mathbf{3} 283$

Physical exercise 3283

Pleural effusion 4348

Posttraumatic stress disorder $\mathbf{1} 66$

Pregnancy 2167

Prevalence 166

Prognosis 4330

Progression 4330

Prognostic factors $\mathbf{6} 564$

Prostate Cancer 2 175, 4330

PSA 4330

Pseudo-monoclonality 5477

PTSD 166

Quality assurance 6535

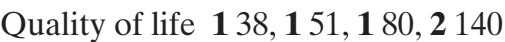

Radiochemotherapy $\mathbf{1} 12$

Radiogenic complications 4334

Radiosensitisation 3295

Radiotherapy 138, 4 325, 6 557, 6564

Raltitrexed 3255

Randomised clinical trials, equipoise 6524

Rectal cancer 5469

Recurrence 2120

Relapse 6573

Resection margins 3218

Response assessment $\mathbf{5} 489$

r-HuEPO 180

Rituximab 4351
Sampling error 132

scintimammography $\mathbf{1} 27$

Second-line treatment $\mathbf{2}$ 161, 3255

Sentinel lymph node biopsy $\mathbf{5} 456$

Sentinel lymph node $\mathbf{5} 456$

Sentinel node 3218

Sézary syndrome 4366

Short-time splenic irradiation 121

Sodium folinate $\mathbf{5} 469$

Soft tissue sarcoma, prognosis 3261

Splenomegaly 121

Sport 3283

Stage 6564

Stem cell transplantation $\mathbf{5} 477$

Study inclusion 5494

Surgery 3218,5446

Surgical margins 4330

Surgical staging 5429

Surveillance $\mathbf{3} 241, \mathbf{3} 303$

Survival 138, 80, 3261

Systemic therapy $\mathbf{3} 234$

Taxol $^{\circledR} \mathbf{2} 128$

Tc-99m sestamibi 127

T-cell lymphomas, cutaneous $\mathbf{4} 366$

T-cell receptor rearrangement $\mathbf{5} 477$

Testicular carcinoma $\mathbf{2} 155$

Thermoradiotherapy 3295

Thrombocytopathia $\mathbf{3} 272$

Thrombocytopenia $\mathbf{3} 272$

Transfusion 173

Transplantation $\mathbf{4} 373$

Trastuzumab $\mathbf{2} 128$

Treatment concepts $\mathbf{2} 167$

Tumor marker $\mathbf{5} 462$

Ultrasound $\mathbf{1} 58$

Ureteral stenosis $\mathbf{4 3 3 4}$

Urokinase $\mathbf{5} 438$

Vaccination 3 234, 5477

Vulvar carcinoma 6564

Weekly therapy $\mathbf{2} 128$

Workflow models 5494

X-ray diagnostics 3241

Xeloda ${ }^{\circledR}, 6578$ 